\title{
Molecular simulations of heterogeneous ice nucleation. I. Controlling ice nucleation through surface hydrophilicity
}

Stephen J. Cox, Shawn M. Kathmann, Ben Slater, and Angelos Michaelides'

Citation: The Journal of Chemical Physics 142, 184704 (2015); doi: 10.1063/1.4919714

View online: http://dx.doi.org/10.1063/1.4919714

View Table of Contents: http://aip.scitation.org/toc/jcp/142/18

Published by the American Institute of Physics

\section{Articles you may be interested in}

Molecular simulations of heterogeneous ice nucleation. II. Peeling back the layers

The Journal of Chemical Physics 142, 184705184705 (2015); 10.1063/1.4919715

The surface charge distribution affects the ice nucleating efficiency of silver iodide

The Journal of Chemical Physics 145, 211924211924 (2016); 10.1063/1.4966018

Pre-ordering of interfacial water in the pathway of heterogeneous ice nucleation does not lead to a two-step crystallization mechanism

The Journal of Chemical Physics 145, 211910211910 (2016); 10.1063/1.4961652

Ice formation on kaolinite: Insights from molecular dynamics simulations

The Journal of Chemical Physics 145, 211927211927 (2016); 10.1063/1.4968796

Heterogeneous nucleation from a supercooled ionic liquid on a carbon surface

The Journal of Chemical Physics 145, 211919211919 (2016); 10.1063/1.4963336

Crystal nucleation as the ordering of multiple order parameters

The Journal of Chemical Physics 145, 211801211801 (2016); 10.1063/1.4962166

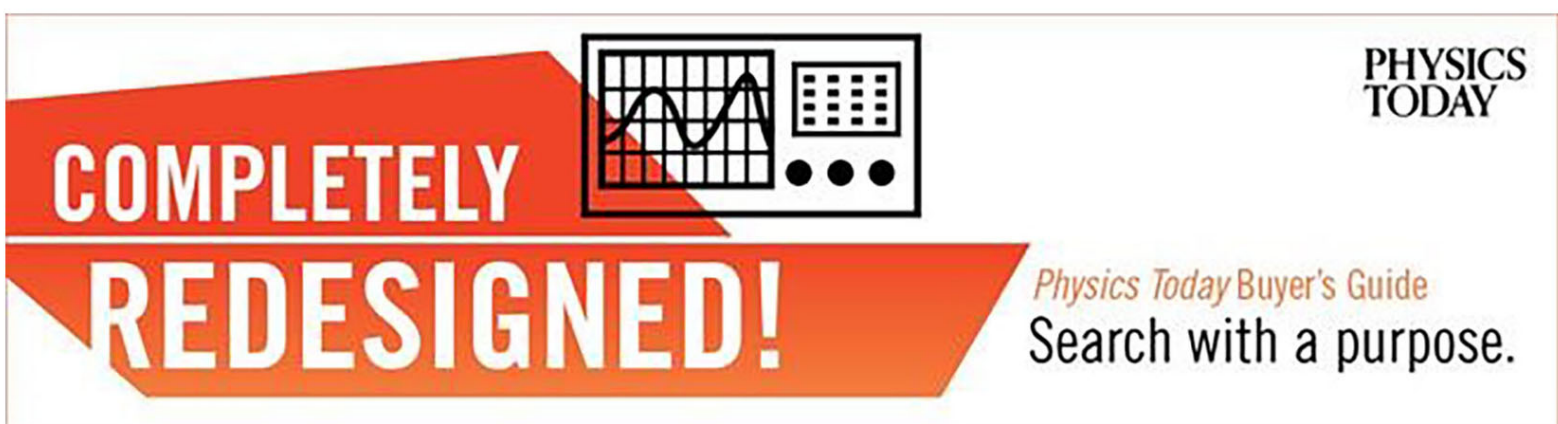




\title{
Molecular simulations of heterogeneous ice nucleation. I. Controlling ice nucleation through surface hydrophilicity
}

\author{
Stephen J. Cox, ${ }^{1,2}$ Shawn M. Kathmann, ${ }^{3}$ Ben Slater, ${ }^{1}$ and Angelos Michaelides ${ }^{1,2, a)}$ \\ ${ }^{1}$ Thomas Young Centre and Department of Chemistry, University College London, 20 Gordon Street, \\ London WC1H OAJ, United Kingdom \\ ${ }^{2}$ London Centre for Nanotechnology, 17-19 Gordon Street, London WC1H OAH, United Kingdom \\ ${ }^{3}$ Physical Sciences Division, Pacific Northwest National Laboratory, Richland, Washington 99352, USA
}

(Received 8 January 2015; accepted 22 April 2015; published online 12 May 2015)

\begin{abstract}
Ice formation is one of the most common and important processes on earth and almost always occurs at the surface of a material. A basic understanding of how the physicochemical properties of a material's surface affect its ability to form ice has remained elusive. Here, we use molecular dynamics simulations to directly probe heterogeneous ice nucleation at a hexagonal surface of a nanoparticle of varying hydrophilicity. Surprisingly, we find that structurally identical surfaces can both inhibit and promote ice formation and analogous to a chemical catalyst, it is found that an optimal interaction between the surface and the water exists for promoting ice nucleation. We use our microscopic understanding of the mechanism to design a modified surface in silico with enhanced ice nucleating ability. (C) 2015 Author(s). All article content, except where otherwise noted, is licensed under a Creative Commons Attribution 3.0 Unported License. [http://dx.doi.org/10.1063/1.4919714]
\end{abstract}

Upon cooling, liquid water crystallizes into solid ice. Due to the presence of a free energy barrier separating the liquid and crystalline states, however, it is possible for liquid water to remain in a metastable "supercooled" state to temperatures far below the equilibrium melting temperature. Heterogeneous ice nucleation, that is, ice nucleation in the presence of impurity particles such as mineral dust, soot, or certain types of bacteria, generally increases the rate of ice nucleation and is the dominant process by which ice forms in nature. ${ }^{1}$ Recent work has argued that any ice formation at temperatures above $-20{ }^{\circ} \mathrm{C}$ must necessarily occur heterogeneously. ${ }^{2}$ Empirically, a large variance in the propensity of different materials to nucleate ice is observed, and due to the importance of ice formation in, e.g., the climate sciences, much effort has been expended in identifying and cataloging the effectiveness of different materials to nucleate ice. ${ }^{1}$ This has motivated many simulation studies of heterogeneous ice nucleation in the presence of different surfaces, including graphite, ${ }^{3,4}$ kaolinite, ${ }^{5,6}$ and silver iodide. ${ }^{7,8}$ Despite the vast amount of research into heterogeneous ice nucleation, major gaps in our knowledge still exist, especially with regard to our understanding of the underlying chemical physics; this is reflected in our inability to accurately predict a material's ice nucleating efficiency and to answer seemingly simple questions such as how does hydrophilicity affect the ice nucleation rate? Not only is an understanding of the chemical physics of heterogeneous ice nucleation needed to predict the ice nucleating efficiency of existing materials ${ }^{9,10}$ but it is also paramount for the rational design of new materials to either promote or inhibit ice nucleation. Controlling ice formation is desirable in a variety of fields, for example, in the cryopreservation of cells, tissues, and organs, ${ }^{11}$ the food and transport industries, and even as a potential means for climate control. ${ }^{12,13}$

\footnotetext{
a)Electronic mail: angelos.michaelides@ucl.ac.uk
}

In contrast to fields such as chemical catalysis ${ }^{14}$ and materials design, ${ }^{15}$ there is currently no comprehensive set of design principles in terms of molecular "descriptors" for making new substances to control ice formation. Put more simply, we do not know which are the relevant microscopic properties of a material that determine its macroscopic ice nucleating efficiency. Often, the so-called "requirements" for a good ice nucleating agent (INA) have been discussed, such as the requirement for a good crystallographic match to ice and the ability of water to chemically bond to the surface of the particle (i.e., hydrophilicity). ${ }^{16}$ Although properties such as a good crystallographic match are important in heterogeneous nucleation of some systems, ${ }^{17,18}$ such criteria have neither served as a full set of guidelines to identify good INAs ${ }^{1}$ nor have they aided the systematic improvement of ice nucleation inhibitors or promoters. Experimentally, there is a disagreement regarding the role of hydrophilicity. For example, Alizadeh et al. ${ }^{19}$ have found ice nucleation to be slower on superhydrophobic surfaces, which they attribute not only to a lower contact area between the water and the surface but also to a larger free energy barrier to nucleation. In contrast, Li et al. ${ }^{20,21}$ found ice nucleation to be enhanced at hydrophobic modified silicon wafers relative to their unmodified hydrophilic counterparts, which was attributed to a faster dynamics of water at the hydrophobic interface. Recently, Lupi et al. investigated the role of hydrophilicity of graphitic surfaces using molecular dynamics simulation: ${ }^{3,4}$ by varying the hydrophilicity in different ways (by uniformly changing the interaction of water with the surface or by introducing hydrophilic species at the surface), they found that the ice nucleating efficacy of the surface could either increase or decrease. Also, it is found on kaolinite (a known hydrophilic INA) and platinum ${ }^{22}$ that the most stable water overlayer can inhibit the growth of subsequent water layers. ${ }^{23,24}$ Furthermore, in the case of requiring 
a good crystallographic match, evidence for ice-like structures at surfaces is in general lacking ${ }^{25}$ and there are also instances where materials with a good crystallographic match to ice are poor INAs ${ }^{26,27}$ (we note that recent simulation studies ${ }^{7,8}$ have found the unreconstructed basal face of silver iodide to act as a template for ice). We are therefore either faced with the prospect of relying on experiments to determine the efficacy of INAs on a case-by-case basis or we can try and rationalize their behavior by elucidating the underlying molecular processes that control heterogeneous ice nucleation.

Here, in the first of a series of two articles, we present results from molecular dynamics (MD) simulations where we directly probe heterogeneous ice nucleation in the presence of a face centered cubic (FCC) nanoparticle (NP). The NP exposes its hexagonal (111) surface as its principal facet and can therefore act as a template for the hexagonal basal face of ice. With this NP completely immersed in water, shown in Fig. 1(a), we perform a series of studies in which we systematically explore the dependence of the nucleation rate on the hydrophilicity of the NP. By comparing these results to reference simulations of homogeneous nucleation, we find a very interesting dependence of the nucleation rate on NP hydrophilicity; the NP can both promote and inhibit ice nucleation and exhibits a maximum nucleation rate at intermediate interaction strengths with the water. By examining the molecular level details of the nucleation processes at different hydrophilicities of the NP, we find that the structure in the immediate vicinity of the interface couples strongly with the nucleation rate. We then use this understanding of the underlying chemical physics to design an improved INA. This first article emphasizes how we can use our microscopic understanding to control ice nucleation. In the second article, we discuss certain aspects of the mechanism in greater detail, as well as contextualizing this work with respect to previous studies on surface hydrophilicity and ice nucleation.

We have used the single site $\mathrm{mW}$ potential to model the interactions between water molecules ${ }^{28}$ which allows us to investigate length and time scales inaccessible to ice nucleation simulations that employ more traditional empirical potentials. ${ }^{5}$ The NP was modeled as a FCC crystal with a lattice constant of $0.392 \mathrm{~nm}$, consisting of 380 atoms. Previous work has suggested that such a lattice may aid in structuring water into ice-like arrangements. ${ }^{29}$ The FCC NP was hemispherical and exposed its (111) face as its primary facet (approximately $2.5 \mathrm{~nm}$ in diameter). For the interaction between the NP atoms and the water molecules, the Lennard-Jones potential $U(r)$ $=4 \epsilon\left[(\sigma / r)^{12}-(\sigma / r)^{6}\right]$ was used, where $r$ is the distance between a NP atom and a water molecule. The hydrophilicity of the NP was controlled by varying $\epsilon$ (a constant value of $\sigma=0.234 \mathrm{~nm}$ was used throughout). As mentioned earlier, Lupi et al. ${ }^{3,4}$ controlled the hydrophilicity of graphitic surfaces not only in this manner but also by introducing hydrophilic species at the surface and found opposite trends: we discuss the possible causes of this apparent discrepancy in more detail in our second paper. ${ }^{42}$ Interactions were truncated after $0.753 \mathrm{~nm}$. This setup yielded contact layers at a height between 0.2 and $0.25 \mathrm{~nm}$ above the (111) surface of the NP, which is in reasonable agreement with values obtained from density functional theory calculations of water at metal surfaces. ${ }^{30,31}$

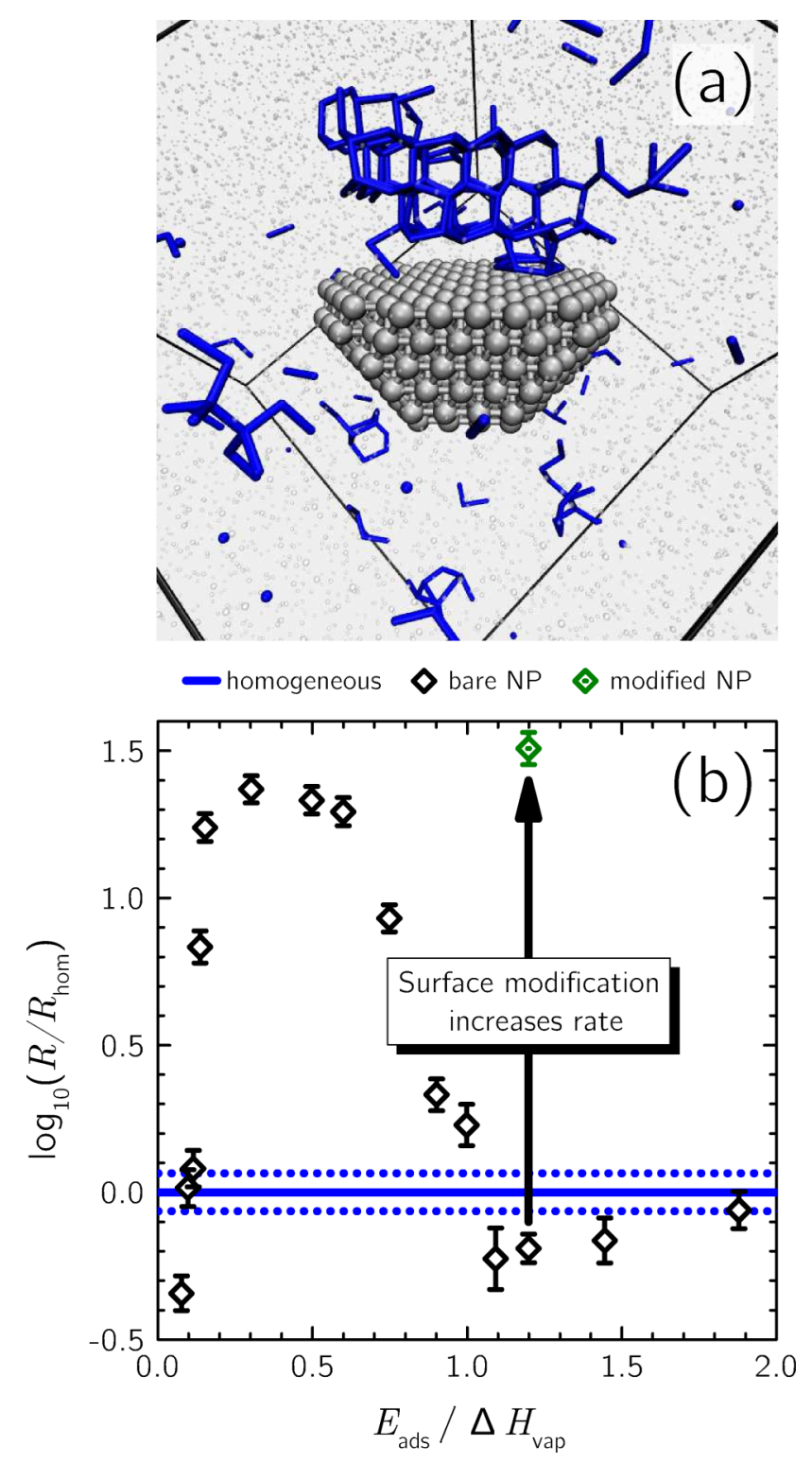

FIG. 1. (a) Snapshot of a typical ice nucleation event on the NP. Ice-like molecules are colored blue and the NP is colored silver. The NP is totally immersed in water (liquid-like molecules are shown by gray dots). (b) Variation of the nucleation rate with the strength of the water-NP interaction $E_{\text {ads. }}$ As $E_{\text {ads }}$ increases, so too does the hydrophilicity. The solid blue line indicates homogeneous nucleation (the dotted lines are an error estimate): data above and below this line indicate promotion and inhibition of ice nucleation by the NP, respectively. It can be seen that at weak and strong water-NP interaction strengths, the NP inhibits nucleation, while at intermediate interaction strengths $\left(E_{\mathrm{ads}} / \Delta H_{\mathrm{vap}} \approx 0.15-0.6\right)$, the NP strongly promotes nucleation.

We must emphasize, however, that we are using simplified model surfaces in order to understand possible general trends that may underlie heterogeneous ice nucleation and that one must exercise caution in trying to make one-to-one correspondences with actual surfaces.

All simulations were performed using the LAMMPS simulation package ${ }^{32}$ with $2944 \mathrm{~mW}$ molecules in a periodic supercell. Previous simulation studies have suggested that the critical ice nucleus varies from $\sim 10$ water molecules at $180 \mathrm{~K}^{33}$ to $\sim 85-265$ at $220 \mathrm{~K}^{34,35}$ giving us confidence that our simulations should not be subjected to serious finite size effects. 
Furthermore, simulations using a slab geometry (approximate dimensions of $66 \AA^{2}$ ) with $4000 \mathrm{~mW}$ molecules confirm that the conclusions drawn from this work are not affected by changes to the box size and shape. For each value of the waterNP interaction energy, $16 \mathrm{MD}$ simulations were performed at $205 \mathrm{~K}$ and 1 bar. Under these conditions, bulk liquid $\mathrm{mW}$ water is still metastable (as opposed to unstable) ${ }^{36}$ but undergoes homogeneous nucleation on a timescale accessible to computer simulation such that statistically meaningful rates can be obtained. To detect "ice-like" molecules, we have used the CHILL algorithm of Moore et al. ${ }^{37}$ (As the CHILL algorithm was designed for bulk homogeneous nucleation, it does not necessarily capture the full behavior in regions of broken symmetry, i.e., interfaces. Nevertheless, it is useful as a qualitative visual aid.) By monitoring the potential energy, we are able to determine the induction time to nucleation for each simulation and thus the probability $P_{\mathrm{liq}}(t)$ that a given system remains liquid after a time $t$ from the start of the simulation. We are able to determine the ice nucleation rate $R$ by fitting $P_{\text {liq }}(t)=\exp \left[-(R t)^{\gamma}\right]$, where $\gamma>0$ is also a fitting parameter. Further details of the fitting procedure and simulation setup are provided in the supplementary material. ${ }^{38}$ In order to gauge the effectiveness of the NP as an INA, we have also studied bulk homogeneous nucleation using identical settings.

Explicit simulations of heterogeneous ice nucleation have only recently started to emerge in the literature (see, e.g., Refs. 3-5, 7, 8, and 39) and to enable a systematic study, we draw conclusions from over 200 successful nucleation trajectories in total. Fig. 1(b) shows the dependence of the nucleation rate on the water surface interaction, the main finding of this study. Specifically, we have plotted $\log _{10}\left(R / R_{\mathrm{hom}}\right)$ vs $E_{\text {ads }} / \Delta H_{\text {vap }}$, where $R_{\text {hom }}$ is the bulk homogeneous rate and $\Delta H_{\text {vap }}$ is the enthalpy of vaporization of bulk $\mathrm{mW}$ water $(10.65 \mathrm{kcal} / \mathrm{mol}$ at $298 \mathrm{~K}){ }^{28} \mathrm{~A}$ rich variety in the ice nucleating behavior is seen: the NP is seen to both promote and, surprisingly, inhibit ice formation. We expect this inhibition effect to be concentration dependent; as the NP concentration becomes more dilute, we expect the rates to tend to that of homogeneous nucleation. At low values of $E_{\text {ads }}$, the heterogeneous nucleation rate is approximately two times lower than $R_{\mathrm{hom}}$. Thus, when the particle is very hydrophobic, it tends to inhibit nucleation. As the water-surface interaction strength increases, so too does the nucleation rate until it reaches a maximum at $E_{\text {ads }} / \Delta H_{\text {vap }} \approx 0.4$ that is nearly 25 times faster than bulk homogeneous nucleation. Beyond the maximum, the rate steadily decreases until $E_{\text {ads }} / \Delta H_{\text {vap }} \approx 1.0$. Further beyond this, the rate remains roughly constant and slightly below $R_{\text {hom }}$.

We now try to understand this intriguing dependence of the nucleation rate on the hydrophilicity of the NP. To this end, we have examined in detail the mechanisms by which nucleation occurred on the NP for the various interaction strengths. As the (111) surface of a FCC crystal exhibits hexagonal symmetry, one possible mechanism for heterogeneous ice nucleation is a template effect whereby the molecules in the contact layer form a hexagonal structure commensurate with the surface. Fig. 2(a) confirms this, where we show a typical ice nucleation event in the presence of the NP with $E_{\text {ads }} / \Delta H_{\text {vap }}$ $\approx 0.3$ (close to the maximum rate). Here, we can clearly see

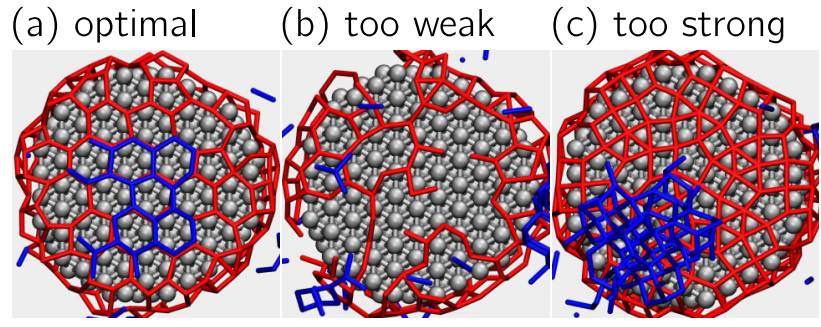

FIG. 2. Sensitivity of the water structure at the surface of the NP to the waterNP interaction strength. (a) Ice nucleation at the NP with $E_{\text {ads }} / \Delta H_{\text {vap }} \approx 0.3$. The water molecules in contact with the NP (colored red) form a hexagonal layer commensurate with the surface that resembles the basal face of ice. The water molecules directly above this contact layer (colored blue) also form a similar hexagonal structure. (b) The structure of water at the NP with $E_{\text {ads }} / \Delta H_{\text {vap }} \approx 0.08$. The water-surface interaction is too weak to stabilize an ice-like structure. (c) The structure of water at the NP with $E_{\text {ads }} / \Delta H_{\text {vap }} \approx 1.2$. The water surface interaction is too strong and the water molecules cannot rearrange into an ice-like configuration. In both (b) and (c), nucleation occurs away from the surface in a homogeneous manner.

that the water molecules in contact with the (111) surface of the NP do indeed form a hexagonal structure commensurate with the surface that resembles the basal face of ice. We can also see that the water molecules directly above this contact layer also form a similar hexagonal structure. The surface is therefore acting to promote ice nucleation by providing an arrangement of adsorption sites that resemble the structure of ice, thereby stabilizing structural fluctuations towards ice-like arrangements in the liquid.

Now that we have established that the NP acts to promote ice nucleation by acting as a template for ice, the dependence of the rate on $E_{\text {ads }}$ can easily be understood as a competition between water-water and water-surface interactions. In Fig. 2(b), we show the structure of water in contact with the NP for $E_{\text {ads }} / \Delta H_{\text {vap }} \approx 0.08$ (the weakest $E_{\text {ads }}$ investigated, which inhibits ice nucleation). Clearly, such a weak water-surface interaction is unable to stabilize ice-like configurations and in fact, ice nucleation is seen to occur away from the surface in a homogeneous manner. Willard and Chandler have found that the structure of the interface between water and a hydrophobic substrate is akin to the liquid-vapor interface $;^{40}$ a recent simulation study of Haji-Akbari et al. ${ }^{41}$ has found that ice nucleation is disfavored at the liquid-vapor interface. This appears to be consistent with our observations and with those of Lupi et al. in Ref. 3. Fig. 2(c), on the other hand, shows the structure of water in contact with the NP for $E_{\text {ads }} / \Delta H_{\text {vap }} \approx 1.2$. While this NP also inhibits ice nucleation, it does so for the opposite reason: the water-surface interaction is too strong, meaning that the water molecules cannot rearrange to form an ice-like layer at the surface. It is also clear that the coverage is higher than when ice forms at the (111) surface, as shown in Fig. 2(a) (we also show this quantitatively in the second paper in this series $^{42}$ ). For this strongly interacting scenario, we also see that ice forms away from the surface in a homogeneous manner. This is also consistent with the observations of Reinhardt and Doye $^{39}$ on ice-like surfaces. The observed coupling between the molecular mechanism and the ice nucleation rate as we change the surface hydrophilicity is actually rather simple; we now demonstrate how we can exploit such simplicity to design a surface with improved ice nucleating efficiency. 
When an ice-like hexagonal overlayer forms at the (111) surface of the NP, such as in Fig. 2(a), it does so with submonolayer coverage, i.e., not all of the available adsorption sites are occupied by water molecules. We refer to these unoccupied adsorption sites on the (111) terrace as "excess" sites. For $E_{\text {ads }} / \Delta H_{\text {vap }} \gtrsim 0.6$, these excess sites are occupied for long times and for nucleation to occur, an area of decreased coverage at the surface must occur such that a hexagonal motif can form. This motif can then act as a template for the hexagonal basal face of ice (a movie showing this for $E_{\text {ads }} / \Delta H_{\text {vap }}$ $\approx 0.9$ is provided ${ }^{38}$ ). When $E_{\text {ads }}>\Delta H_{\text {vap }}$, it becomes favorable for a water molecule to occupy a site on the surface, including the excess sites, rather than a position in the bulk liquid. This prevents the water molecules in the contact layer from forming the hexagonal arrangements required for ice nucleation at this NP. By this rationale, if the density of available adsorption sites was lower, then the template effect (and the enhanced nucleation rate) may be preserved at higher values of $E_{\text {ads }}$. To this end, we have modified the (111) surface of the NP by adsorbing small molecules, at the excess sites, which only have a weak interaction with water, ${ }^{43}$ and recomputed the nucleation rate with $E_{\text {ads }} / \Delta H_{\text {vap }} \approx 1.2$. As seen in Fig. 3, the template effect is indeed recovered. It is also seen in Fig. 1(b) that this modified surface enhances ice nucleation by a factor of 50 compared to the unmodified surface. This is a clear demonstration of how the molecular insight into heterogeneous ice nucleation can be used to rationally design surfaces of different ice nucleating abilities. Experimentally, this could be realized through adsorption of small molecules to the surface (e.g., carbon monoxide) or through surface alloying. In fact, alloying a platinum (111) surface with tin is observed to promote the formation of a hexagonal ice-like bilayer under ultra-high vacuum conditions, ${ }^{45,44}$ in a fashion analogous to

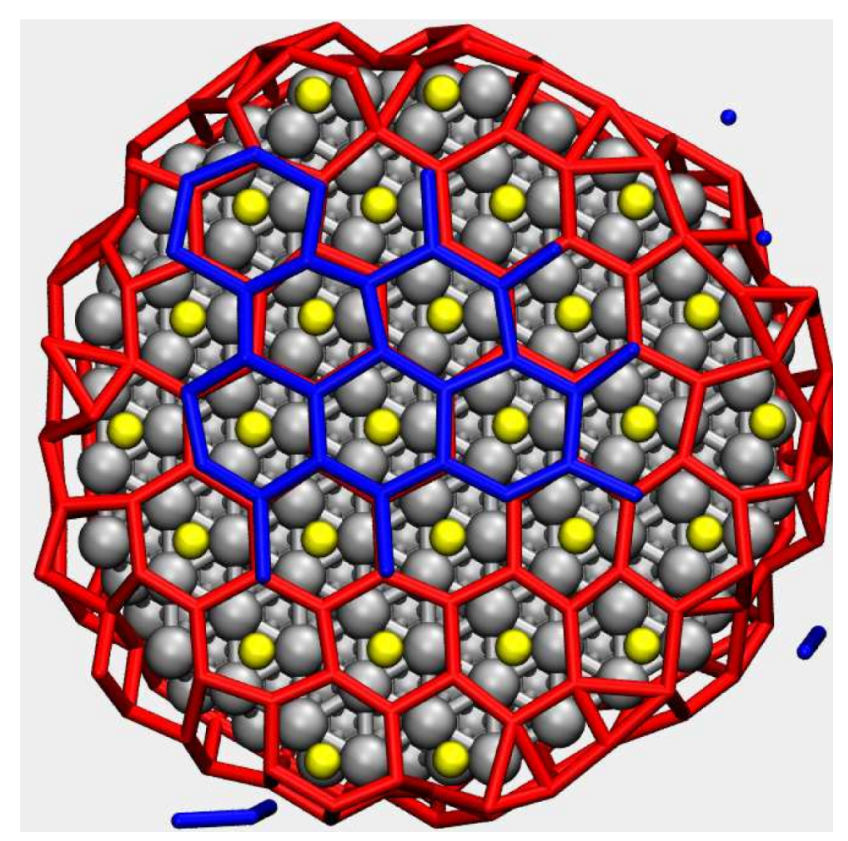

FIG. 3. Surface modification to promote ice nucleation for strong water-NP interaction strengths $\left(E_{\text {ads }} / \Delta H_{\text {vap }}>1.0\right)$. By introducing small adsorbates (colored yellow) at the (111) surface, the template effect can be recovered (cf. Fig. 2(c)). The nucleation rate is increased by approximately a factor 50 compared to the bare NP for $E_{\text {ads }} / \Delta H_{\mathrm{vap}} \approx 1.2$, as indicated in Fig. 1(b). our modified surface (the tin atoms at the surface act in part to reduce the density of adsorption sites). We also note that in our simulations, the nucleation rate can also be decreased by adsorbing small molecules such that the NP can no longer act as a template.

More generally, the sensitivity of the nucleation rate on surface hydrophilicity could be tested by, e.g., using nanoparticles of gold or silica functionalized with organic molecules of varying hydrophobicities. In addition to using well established methods such as the droplet freezing techniques ${ }^{46}$ it may also be possible to exploit recent advances in femtosecond X-ray scattering techniques that have allowed real-time monitoring of homogeneous ice nucleation in micrometer sized water droplets. ${ }^{47}$ Not only could such an experimental protocol be used to compare rates of ice nucleation in the presence of immersed NPs, but information regarding the impact of such NPs on the microscopic structure of the liquid should also be available.

In summary, we have used computer simulations to systematically compare heterogeneous ice nucleation rates in the presence of a simple model nanoparticle of varying hydrophilicities. This complements a number of recent simulation studies on specific systems..$^{3-5,7,8}$ We have seen that the nanoparticle can promote ice nucleation by acting as a template for the hexagonal ice lattice, but that the ice nucleating efficiency is lost if adsorption is too strong, due to a high coverage of water molecules destroying the template effect. Modification of the surface such that the coverage of water molecules is reduced recovers this template effect and enhanced nucleation can be achieved for strongly adsorbing surfaces, clearly demonstrating how molecular level understanding of heterogeneous ice nucleation can be used to manipulate the rate of ice formation. The use of molecular descriptors to predict useful macroscopic properties of materials has been successfully used in other fields, such as chemical catalysis. ${ }^{14}$ Designing new catalysts for reactions such as methanation $\left(\mathrm{CO}+3 \mathrm{H}_{2}\right.$ $\longrightarrow \mathrm{CH}_{4}+\mathrm{H}_{2} \mathrm{O}$ ) has relied upon the establishment of a Sabatier principle based on a computationally tractable quantity (in this case, the dissociation energy of $\mathrm{CO}$ at the surface). ${ }^{48} \mathrm{We}$ have seen that for the surface investigated in this study, the adsorption energy of a single water molecule can be used to describe the heterogeneous ice nucleation rate. Although a comprehensive set of rules still requires further experimental and theoretical investigation, the results presented here suggest that if the surface acts as a template for ice, then one must tune either the density of adsorption sites or the propensity of water to adsorb to the surface. In our second article, ${ }^{42}$ we show that the variation of the ice nucleation rate upon surface hydrophilicity is dependent upon the surface topography, demonstrating that the combined effect of different surface properties needs to be considered when trying to understand what makes a good INA. Other properties such as the crystallographic match to ice and the role of surface defects are also likely to be important, as will more complex interactions such as electrostatics and explicit hydrogen bonding. The results presented in this letter serve as a platform upon which future studies can be conducted.

Dr. Gabriele C. Sosso is thanked for sharing his data from larger simulations in a slab geometry. We are grateful 
to the London Centre for Nanotechnology and UCL Research Computing for computation resources, and the UK's national high performance computing service (from which access was obtained via the UK's Material Chemistry Consortium, EP/ F067496). S.M.K. was supported fully by the U.S. Department of Energy, Office of Basic Energy Sciences, Division of Chemical Sciences, Geosciences \& Biosciences. Pacific Northwest National Laboratory (PNNL) is a multiprogram national laboratory operated for DOE by Battelle. S.J.C. was supported by a student fellowship funded jointly by UCL and BES. A.M. is supported by the European Research Council under the European Union's Seventh Framework Programme (No. FP/2007-2013)/ERC Grant Agreement No. 616121 (HeteroIce project) and the Royal Society through a Royal Society Wolfson Research Merit Award.

${ }^{1}$ B. J. Murray, D. O'Sullivan, J. D. Atkinson, and M. E. Webb, Chem. Soc. Rev. 41, 6519 (2012).

${ }^{2}$ E. Sanz, C. Vega, J. R. Espinosa, R. Caballero-Bernal, J. L. F. Abascal, and C. Valeriani, J. Am. Chem. Soc. 135, 15008 (2013).

${ }^{3}$ L. Lupi, A. Hudait, and V. Molinero, J. Am. Chem. Soc. 136, 3156 (2014).

${ }^{4}$ L. Lupi and V. Molinero, J. Phys. Chem. A 118, 7330 (2014).

${ }^{5}$ S. J. Cox, Z. Raza, S. M. Kathmann, B. Slater, and A. Michaelides, Faraday Discuss. 167, 389 (2013).

${ }^{6}$ T. Croteau, A. K. Bertram, and G. N. Patey, J. Phys. Chem. A 112, 10708 (2008).

${ }^{7}$ S. A. Zielke, A. K. Bertram, and G. N. Patey, "A molecular mechanism of ice nucleation on model AgI surfaces," J. Phys. Chem. B (published online).

${ }^{8}$ G. Fraux and J. P. K. Doye, J. Chem. Phys. 141, 216101 (2014).

${ }^{9}$ T. Bartels-Rausch, Nature 494, 27 (2013).

${ }^{10}$ T. F. Stocker, D. Qin, G. K. Plattner, M. Tignor, S. K. Allen, J. Boschung, A. Nauels, Y. Xia, V. Bex, and P. M. Midgley, Climate Change 2013: The Physical Science Basis: Working Group I Contribution to the Fifth Assessment Report of the Intergovernmental Panel on Climate Change (Cambridge University Press, Cambridge, United Kingdom and New York, NY, USA, 2013).

${ }^{11}$ R. C. Deller, M. Vatish, D. A. Mitchell, and M. I. Gibson, Nat. Commun. 5, 3244 (2014).

${ }^{12}$ T. Storelvmo and N. Herger, J. Geophys. Res.: Atmos. 119, 2375, doi:10.1002/2013jd020816 (2014).

${ }^{13}$ H. Muri, J. E. Kristjánsson, T. Storelvmo, and M. A. Pfeffer, J. Geophys. Res.: Atmos. 119, 4174, doi:10.1002/2013jd021063 (2014).

${ }^{14}$ J. K. Nørskov, T. Bligaard, J. Rossmeisl, and C. H. Christensen, Nat. Chem. 1, 37 (2009).

${ }^{15}$ S. Curtarolo, G. L. Hart, M. B. Nardelli, N. Mingo, S. Sanvito, and O. Levy, Nat. Mater. 12, 191 (2013).

${ }^{16} \mathrm{H}$. R. Pruppacher and J. D. Klett, Microphysics of Clouds and Precipitation: Second Revised and Enlarged Edition with an Introduction to Cloud Chemistry and Cloud Electricity (Kluwer Academic Publishers, Dordrecht, The Netherlands, 1997).

${ }^{17}$ A. Cacciuto and D. Frenkel, Phys. Rev. E 72, 041604 (2005).

${ }^{18}$ G. I. Tóth, G. Tegze, T. Pusztai, and L. Gránásy, Phys. Rev. Lett. 108, 025502 (2012).
${ }^{19}$ A. Alizadeh, M. Yamada, R. Li, W. Shang, S. Otta, S. Zhong, L. Ge, A. Dhinojwala, K. R. Conway, V. Bahadur, A. J. Vinciquerra, B. Stephens, and M. L. Blohm, Langmuir 28, 3180 (2012).

${ }^{20}$ K. Li, S. Xu, W. Shi, M. He, H. Li, S. Li, X. Zhou, J. Wang, and Y. Song, Langmuir 28, 10749 (2012).

${ }^{21} \mathrm{~K}$. Li, S. Xu, J. Chen, Q. Zhang, Y. Zhang, D. Cui, X. Zhou, J. Wang, and Y. Song, Appl. Phys. Lett. 104, 101605 (2014).

${ }^{22}$ G. A. Kimmel, N. G. Petrik, Z. Dohnálek, and B. D. Kay, Phys. Rev. Lett. 95, 166102 (2005).

${ }^{23}$ X. L. Hu and A. Michaelides, Surf. Sci. 601, 5378 (2007).

${ }^{24}$ X. L. Hu and A. Michaelides, Surf. Sci. 602, 960 (2008).

${ }^{25}$ J. Carrasco, A. Hodgson, and A. Michaelides, Nat. Mater. 11, 667 (2012).

${ }^{26}$ V. Sadtchenko, G. E. Ewing, D. R. Nutt, and A. J. Stone, Langmuir 18, 4632 (2002).

${ }^{27}$ P. Conrad, G. E. Ewing, R. L. Karlinsey, and V. Sadtchenko, J. Chem. Phys. 122, 064709 (2005)

${ }^{28}$ V. Molinero and E. B. Moore, J. Phys. Chem. B 113, 4008 (2009).

${ }^{29}$ S. J. Cox, S. M. Kathmann, J. A. Purton, M. J. Gillan, and A. Michaelides, Phys. Chem. Chem. Phys. 14, 7944 (2012).

${ }^{30}$ A. Michaelides, V. A. Ranea, P. L. de Andres, and D. A. King, Phys. Rev. Lett. 90, 216102 (2003).

${ }^{31}$ J. Carrasco, J. Klimeš, and A. Michaelides, J. Chem. Phys. 138, 024708 (2013).

${ }^{32}$ S. Plimpton, J. Comput. Phys. 117, 1 (1995).

${ }^{33}$ E. B. Moore and V. Molinero, Phys. Chem. Chem. Phys. 13, 20008 (2011).

${ }^{34}$ A. Reinhardt and J. P. K. Doye, J. Chem. Phys. 136, 054501 (2012).

${ }^{35}$ T. Li, D. Donadio, G. Russo, and G. Galli, Phys. Chem. Chem. Phys. 13, 19807 (2011).

${ }^{36}$ E. B. Moore and V. Molinero, Nature 479, 506 (2011).

${ }^{37}$ E. B. Moore, E. de la Llave, K. Welke, D. A. Scherlis, and V. Molinero, Phys, Chem. Chem. Phys. 12, 4124 (2010).

${ }^{38}$ See supplementary material at http://dx.doi.org/10.1063/1.4919714 for additional details regarding the simulation methods and the data fitting procedure.

${ }^{39}$ A. Reinhardt and J. P. K. Doye, J. Chem. Phys. 141, 084501 (2014).

${ }^{40}$ A. P. Willard and D. Chandler, J. Chem. Phys. 141, 18C519 (2014).

${ }^{41}$ A. Haji-Akbari, R. S. DeFever, S. Sarupria, and P. G. Debenedetti, Phys. Chem. Chem. Phys. 16, 25916 (2014).

${ }^{42}$ S. J. Cox, S. M. Kathmann, B. Slater, and A. Michaelides, J. Chem. Phys. 142, 184705 (2015).

${ }^{43}$ The small adsorbates were modeled using Lennard-Jones spheres, with $\epsilon=0.18 \mathrm{kcal} / \mathrm{mol}$ and $\sigma=0.18 \mathrm{~nm}$.

${ }^{44}$ F. McBride, G. R. Darling, K. Pussi, and A. Hodgson, Phys. Rev. Lett. 106, 226101 (2011).

${ }^{45}$ S. Nie, P. J. Feibelman, N. C. Bartelt, and K. Thürmer, Phys. Rev. Lett. 105, 026102 (2010).

${ }^{46}$ J. D. Atkinson, B. J. Murray, M. T. Woodhouse, T. F. Whale, K. J. Baustian, K. S. Carslaw, S. Dobbie, D. O'Sullivan, and T. L. Malkin, Nature 498, 355 (2013).

${ }^{47}$ J. A. Sellberg, C. Huang, T. A. McQueen, N. D. Loh, H. Laksmono, D. Schlesinger, R. G. Sierra, D. Nordlund, C. Y. Hampton, D. Starodub, D. P. DePonte, M. Beye, C. Chen, A. V. Martin, A. Barty, K. T. Wikfeldt, T. M. Weiss, C. Caronna, J. Feldkamp, L. B. Skinner, M. M. Seibert, M. Messerschmidt, G. J. Williams, S. Boutet, L. G. M. Pettersson, M. J. Bogan, and A. Nilsson, Nature 510, 381 (2014).

${ }^{48}$ M. P. Andersson, T. Bligaard, A. Kustov, K. E. Larsen, J. Greeley, T. Johannessen, C. H. Christensen, and J. K. Nørskov, J. Catal. 239, 501 (2006). 